Results. All 41 patients reported a lifetime burden with schizophrenia adversely impacting employment, relationships, emotional health, social activities, and daily tasks. Hospitalization for schizophrenia management was another reported aspect of disease burden. Although most $(n=32)$ patients reported previous medication benefits, side effects affecting physical, emotional/behavioral, and cognitive functioning were reported by all $(n=41)$. Following OLZ/SAM treatment, 39/41 patients (95\%) reported improvements in symptoms including hallucinations, paranoia, depression, sleep, and concentration. Furthermore, patients described improvements in self-esteem, social activities, relationships, and daily activities. Twenty-three patients (56\%) reported side effects attributed to OLZ/SAM; lack of energy $(\mathrm{n}=12[29 \%])$ and dry mouth $(\mathrm{n}=5$ [12\%]) were most common. Twenty-four (59\%) patients were "very satisfied" with OLZ/SAM; most $(\mathrm{n}=35[85 \%])$ preferred to continue OLZ/SAM vs switching to another medication. As most substudy patients $(\mathrm{n}=40 ; 98 \%)$ completed the extension study, satisfied patients may be overrepresented in this analysis.

Conclusion. This qualitative interview approach provided valuable insight into patients' experiences with previous medications and OLZ/SAM. Overall, most patients reported treatment satisfaction and improvements in symptoms, function, and healthrelated quality of life with OLZ/SAM.

Funding. Alkermes, Inc. by demographic characteristics. Case-control comorbidity comparisons were performed using prevalence rate ratios (PRRs) and 95\% CIs. Per-database medication exposure ( $=1$ National Drug Code in outpatients grouped by Redbook classification) was also assessed.

Results. Schizophrenia prevalence was $0.11 \%$ and $0.99 \%$ in commercially and Medicaid-insured patients, respectively. In both databases, comorbidity prevalence was higher among schizophrenia cases versus controls in approximately $=80 \%$ of the CCS level 2 categories assessed. Common top categories of comorbidities for schizophrenia cases were mood disorders, anxiety disorders, other connective tissue disease, and diseases of the heart. Comorbidities with the highest case-control PRRs included personality disorders, suicide and intentional self-inflicted injury, and impulse control disorders. Across databases, the most commonly prescribed medications in cases were antipsychotics, antidepressants, and analgesics/antipyretics opiate agonists; the most highly prescribed antipsychotics were risperidone, quetiapine, aripiprazole, and olanzapine.

Conclusions. This large-scale analysis quantifies the high prevalence of medical and psychiatric comorbidity burden in patients with schizophrenia, highlighting the importance of integrated medical and psychiatric care.

Funding. Alkermes, Inc.

\section{Disease Prevalence, Comorbid Conditions, and Medication Utilization Among Patients with Schizophrenia in the United States}

\section{Brittany Roy, $\mathrm{MPH}^{1}$, Ankitaben Shah ${ }^{1}$, Gary Bloomgren, $\mathrm{MD}^{1}$, Made Wenten, $\mathrm{PhD}, \mathrm{MPH}^{1}$, Jianheng Li, $\mathrm{MPH}^{2}$ and Cathy Lally, $\mathrm{MSPH}^{2}$}

${ }^{1}$ Alkermes, Inc., Waltham, MA, USA, and ${ }^{2}$ Epidemiologic Research \& Methods, LLC,

Presenting Author: Brittany Roy Atlanta, GA, USA

\section{Long-Term Safety and Efficacy of Deutetrabenazine in Younger and Older Patients With Tardive Dyskinesia}

Martha Sajatovic, $\mathrm{MD}^{1}$, Amanda Wilhelm, $\mathrm{PhD}^{2}$, Stacy Finkbeiner, $\mathrm{PhD}^{2}$, Hadas Barkay, $\mathrm{PhD}^{3}$, Nayla Chaijale, $\mathrm{PhD}^{2}$, Nicholas Gross, $\mathrm{MS}^{2}$ and Mark Forrest Gordon, MD ${ }^{2}$

${ }^{1}$ University Hospitals Cleveland Medical Center, Case Western Reserve University School of Medicine, Cleveland, OH, USA, ${ }^{2}$ Teva Pharmaceutical Industries Ltd., West Chester, PA, USA, and ${ }^{3}$ Teva Pharmaceutical Industries Ltd., Netanya, Israel

Presenting Author: Stacy Finkbeiner

\begin{abstract}
Objective. Disease prevalence, comorbid conditions, and pharmacological treatments were examined in a large population of US commercial- or Medicaid-insured individuals with schizophrenia.

Methods. This retrospective, cross-sectional claims analysis sourced data from the IBM MarketScan Commercial and Medicare Supplemental Databases and the Multi-state Medicaid Database (01Jan2009 to 30Jun2016). Cases were defined by $=1$ diagnostic claim (ICD-9-CM/ICD-10-CM) for schizophrenia during the study period. Comorbidities (=1 ICD-9-CM/ICD-10-CM diagnosis code) were grouped according to Clinical Classifications Software (CCS) level 2 categories. For the per-database analysis of comorbidities, schizophrenia cases were matched with controls
\end{abstract}

\begin{abstract}
Background. Tardive dyskinesia (TD) is an involuntary movement disorder that is more prevalent in older patients. However, there is limited information on TD treatment for this population. In two 12-week pivotal trials (ARM-TD and AIM-TD), TD patients demonstrated significant improvements in Abnormal Involuntary Movement Scale (AIMS) score with deutetrabenazine versus placebo.

Methods. Patients who completed ARM-TD or AIM-TD enrolled in an open-label extension (OLE) study. This post hoc analysis assessed change and percent change from baseline in AIMS score, response rates for $\geq 50 \%$ AIMS improvement, Patient Global Impression of Change (PGIC), Clinical Global
\end{abstract}

